\title{
Stabilizing the Uniform Lying Helix Alignment in Chiral Nematic Liquid \\ Crystals Using Direct Laser Writing
}

\author{
Chloe C. Tartan, Patrick. S Salter, Martin J. Booth, Stephen M. Morris and Steve J. Elston \\ Department of Engineering Science, University of Oxford, Parks Road, Oxford, OX1 3PJ, United \\ Kingdom
}

Shortened Title: DirectLaserWritingforULHAlignment

\begin{abstract}
We investigate a novel approach for controlling the alignment of a fast-switching chiral nematic liquid crystal state using a high resolution two-photon absorption laser scanning lithography technique with aberration correction that permits the engineering of photonic structures in-situ. Walls of polymer network are engineered parallel and perpendicular to the helical axis of a uniform lying helix in chiral nematic liquid crystals in order to stabilize the alignment in the absence of an electric field and enhance the flexo-electro-optic response.
\end{abstract}

\section{INTRODUCTION}

Nematic liquid crystals (LCs) are complex anisotropic fluids that have long-range orientational order and are thermodynamically stable between the temperature range of crystalline solids and isotropic liquids. The birefringent nature of these dielectric liquid crystalline materials results in electro-optic properties that have been exploited most famously in the displays industry. However, addressing the stability of more complex chiral nematic LC states can lead to a wider range of photonic applications. 
The chiral flexo-electro-optic (FEO) effect can be observed when a short pitch chiral nematic is aligned in a uniform lying helix (ULH) as illustrated in the schematic of Figure 1, so that the chiral nematic has its helical axis aligned uniformly between two substrates. Under the application of an electric field perpendicular to the helix axis, an in-plane tilt $(\Phi)$ is induced in the optical axis that couples to the flexoelectric coefficients,

$$
\tan \Phi=e p /(2 \pi k) \mathbf{E}
$$

where $p$ is the pitch, $k=\left(k_{1}+k_{3}\right) / 2$ is the average elastic constant and $e=\left(e_{1}-e_{3}\right) / 2$ is the effective flexoelectric coefficient.

In the linear regime of the FEO effect, short pitch cholesterics lead to fast changes in the optical state and sub-millisecond response times in liquid crystals. However, the FEO effect is limited by the dielectric coupling that tends to unwind the helix [1]. Unfortunately the ULH state is incompatible with strong planar and homeotropic alignments, becoming unstable when the chiral nematic is confined in a cell with spatially uniform aligning surfaces.

Alternatively, under the application of a moderate electric field, a chiral nematic sample with a positive dielectric anisotropy can be cooled from the isotropic phase into a ULH. However, upon removal of the electric field for a planar-aligned device, the ULH relaxes back to the lower energy Grandjean state, whereby the helix axis is aligned perpendicular to the substrates. Periodic (horizontal/vertical) anchoring conditions have also been shown to stabilize the ULH texture so long as an external electric field is maintained [3]. 
In order for the ULH to be locked in, a set of defects or periodic distortions can be introduced at the chiral nematic-substrate interface. Komitov et al. [4] reported the fabrication of periodic anchoring conditions using hard-contact photolithography to pattern a photoresist and consequently were able to stabilize the alignment in the presence of an applied electric field.

Weak homeotropic alignment results in a lower energy of the ULH relative to the Grandjean texture. However, the rotational symmetry at the substrate causes the alignment to collapse into domains due to the lack of a preferred direction for the helix to form. Carbone et al. [2] have exploited the stable, low energy ULH texture that occurs at the homeotropic substrate by breaking its rotational symmetry using two-photon absorption (TPA) laser scanning lithography to form periodic surface relief structures with a periodicity equal to the pitch of the bulk chiral nematic. The authors report electro-optic observations that reveal stable alignment even in the absence of an electric field. They also report contrast ratios that are not sufficient for commercial applications. The same group tried another approach based on a holographic technique to engineer POLYCRIPS microchannels that consist of roughly equal areas of alternating polymer and LC slices. [5]. However, curing large regions inhibits the switching of the LC molecules thereby reducing the intensity modulation resultant from the FEO effect.

Following on from the previous work based upon polymer stabilization, we employ a high resolution TPA laser scanning lithography technique to engineer polymer network structures of various sizes and orientations that can control the ULH alignment. Walls of polymer network have the potential to enhance the observed flexoelectric switching, relative to bulk polymer networks that can inhibit the electro-optic effect. Polymeric networks can be 
engineered either parallel to the helical axis, following on from the POLYCRIPS microchannels, or perpendicular to the helix following on from the periodic distortions described above.

\section{EXPERIMENTAL}

The commercial eutectic nematic E7, with a birefringence of $\Delta \mathrm{n}=0.165$ and a positive dielectric anisotropy of $\Delta \varepsilon=10.37$, was used as the host liquid crystal. The chiral nematic mixture consisted of $4.1 \mathrm{wt} \%$ of a high twisting power chiral dopant (BDH1281, Merck), 26.8 wt \% monomer (RM257, Merck) and $0.6 \mathrm{wt} \%$ photoinitiator (IRG819, Ciba) and was mixed at $80^{\circ} \mathrm{C}$ for a few hours. A homeotropic device of thickness $5 \mu \mathrm{m}$ was capillary filled with the resulting chiral nematic liquid crystal monomer mixture in the isotropic phase.

A two photon process is employed in order to achieve submicron resolution in the fabrication of the polymer walls in order to control the alignment of the ULH. The light source is a Titanium-Sapphire oscillator laser with a wavelength of $790 \mathrm{~nm}$ (Newport Mai Tai), a power of $300 \mathrm{~mW}$, and an $80 \mathrm{fs}$ pulse duration giving a pulse repetition frequency of $80 \mathrm{MHz}$. The nonlinear nature of the photopolymerization process helps to minimize the amount of exposure to the sample and the possibility for polymerization in the bulk.

The optical set-up for the two photon photopolymerization process is illustrated in Figure 2. The laser beam is transmitted through a half wave-plate and polarizer for power control, before being expanded onto an adaptive optics element, a liquid crystal spatial light modulator (SLM). Phase patterns can be displayed on the SLM to correct for aberrations due to the refractive index mismatch as light passes through the liquid crystal and the substrate, making it 
possible to engineer polymeric photonic structures in-situ. Through aberration correction, it becomes possible to use high numerical aperture (NA) objectives for the photopolymerization leading to enhanced resolution in the fabricated walls [6]. In practice, the SLM is mapped onto the pupil plane of the objective in a $4 \mathrm{f}$ configuration. The desired phase pattern to be displayed on the SLM is superimposed on a blazed phase grating and the first order diffracted light from the grating region used for the fabrication. A pinhole placed in the Fourier plane of the SLM blocks all light except that incident on the grating region. Thus by controlling the size, modulation depth and phase of the grating region, complete control is gained over the amplitude and phase of the light in the pupil plane of the objective [7].

The sample was mounted on a xyz-piezoelectric translation stage for fine positioning and a built-in polarizing microscope was used for inspection during fabrication. The objective was a 20x Zeiss lens with a numerical aperture of 0.5 and a working distance of $2 \mathrm{~mm}$. Polymeric walls with periodicities of the order of the device thickness were written using the full power Mai Tai laser across two regions of $0.2 \mathrm{~mm}$ by $1 \mathrm{~mm}$. The optimum exposure time that dictates the thickness of the polymer walls was established at a writing speed of $150 \mu \mathrm{m} / \mathrm{s}$. The polymer structures were engineered after a ULH had been formed by a process of annealing under the application of a $\pm 20 \mathrm{~V}, 1 \mathrm{kHz}$ square wave. Mechanically shearing the sample whilst gradually cooling to room temperature from the isotropic phase improves the ULH alignment on account of shear flow in the liquid crystal. Upon cooling the sample to room temperature, polymeric walls were engineered parallel and perpendicular to the helical axis, whilst maintaining the applied electric field. 


\section{RESULTS AND DISCUSSION}

Optical microscope images of the periodic polymer walls were taken between crossed polarisers when the optical axis of the device was angled at $0^{\circ}$ (optical extinction) and $45^{\circ}$ (maximum transmission) to the analyser. Figure 3 (a) shows the dark and bright states of the device when the polymer walls are written parallel to the helical axis, while Figure 3 (b) illustrates the ULH alignment achieved when polymer network structures are formed perpendicular to the helix. Both set of images show reasonable ULH alignment, although there is slightly better contrast between the dark and bright states for the case shown in Figure 3 (a).

During the annealing process, the ULH did not form uniformly across the device, despite improvements from shear flow, due to the degeneracy of the homeotropic anchoring as there is no preferential orientation. Consequently, some regions between the polymer walls are not exactly parallel or perpendicular to the helix. Interestingly, a region in between the two polymer network structures, as seen in Figure 3 (c), shows very good ULH alignment with better contrast between bright and dark states.

Figures 4 (a) - (c) show the electro-optic responses corresponding to the alignments shown in Figures 3 (a) - (c), respectively, when a $\pm 27.5 \mathrm{~V}, 500 \mathrm{~Hz}$ square wave is applied to the device. Engineering polymer walls parallel and perpendicular to the helical axis produce a similar modulation in the transmission as can be seen in Figures 4 (a) and (b). Both measurements gave a switching angle of $1.1^{\circ}$. The modulation in the transmission detected from the non-polymerized region between the polymer network structures, as shown in Figure 4 (c), is 
almost double that of Figures 4 (a) and (b), due to the fact there is no polymer network there to hinder the switching. Applying mechanical shear to this non-polymerized region revealed the presence of flow, confirming that there was no formation of polymer network. A switching angle of $2.1^{\circ}$ was measured from this region.

It would be interesting to fabricate polymeric walls of different shapes that could confine non-polymerized chiral nematic regions so as to ensure that the magnitude of the electro-optic effect is as large as possible whilst simultaneously achieving a uniform alignment. For practical applications however, a larger tilt angle would be required than those obtained from the test device presented in this paper. It is worth noting that the materials used in this investigation are not optimized to produce large tilt angles. An enhancement of the intensity modulation could therefore be achieved through the use of bimesogenic compounds that are synthesized to give large flexoelastic ratios $(e / k)$ whilst also possessing a low dielectric anisotropy $[8,9]$. Moreover, the use of a different reactive mesogen that itself is flexoelectric for the formation of the polymer networks would also contribute towards the flexoelectric tilt angle in addition to the host liquid crystal.

\section{CONCLUSIONS}

A two-photon absorption laser scanning lithography technique with SLM-based aberration correction has been employed to achieve spontaneous and stable alignment of the uniform lying helix geometry that is necessary to observe the flexoelectro-optic effect in chiral nematic liquid crystals. The alignment was controlled by polymer walls that were written in-situ either parallel or perpendicular to the helical axis, and with periodicities of the order of the device thickness. The ULH is shown to be stable upon removal of the externally applied electric 
field between the polymer network structures, although the alignment is not completely uniform leading to relatively low contrast between the bright and dark states. However, a nonpolymerized region between the two polymer structures engineered parallel and perpendicular to the helix shows good contrast suggesting that alternative configurations of the polymer walls may lead to a more uniform alignment and enhanced electro-optic switching. The submicron resolution process could be extended to fabricate more complex photonic structures or used to control the stability of other interesting electro-optic effects in liquid crystalline materials.

\section{ACKNOWLEDGMENTS}

The authors would like to thank Merck Chemicals Ltd. and the Engineering Physical Sciences Research Council for support of an Industrial CASE Studentship.

\section{REFERENCES}

1. S. T. Lagerwall and B. Stebler, SPIE, 2372, 59, (1995)

2. G. Carbone, D. Corbett, S. J. Elston, P. Raynes, A. Jesacher, R. Simmonds and M. Booth, Mol. Crys. Liq. Cryst., 544: 37/[1025]-49/[1037] (2011)

3. R. B. Meyer and J. S. Patel, US Patent 4917475 (1987)

4. L. Komitov, G. P. Bryan-Brown, E. L. Wood and A. B. J Smout, J. Appl. Phys., 86(7): $3508-3511$ (1999)

5. G. Carbone, P. Salter, S. J. Elston, P. Raynes, L. De Sio, S. Ferjani, G. Strangi, C. Umeton and R. Bartolino, Appl. Phys. Lett., 95, 011102 (2009)

6. M. J. Booth, Phil. Trans. R. Soc. A, 365, 2829-2843 (2007) 
7. P. S. Salter, A. Jesacher, J. B. Spring, B. J. Metcalf, N. Thomas-Peter, R. D. Simmonds, N. K. Langford, I. A. Walmsley and M. J. Booth, Opt. Lett., 37 (4) (2012)

8. H. J. Coles, B. Musgrave, M. J. Coles and J. Willmott, J. Mater. Chem., 11 (2001)

9. A. E. Blatch, M. J. Coles, B. Musgrave, and H. J. Coles, Mol. Crys. Liq. Crys. 401 (2003) 